\title{
Changes in the definition of term infants and their implications in perinatal care. Are they being accomplished?
}

For many years, a pregnancy was considered full term any time between 38 and 41 weeks of gestation. This classification seemed adequate because it took into account a greater maturation of newborns. However, to my understanding, there was no reason to justify, a few decades ago, the definition of term pregnancy as of 37 weeks of gestation. The complexity of this subject requires a broad and honest look, which at least considers the main consequences of such change. I believe it caused no benefits, only damages. One of such damages was the indiscriminate and progressive increase of C-sections at 37 weeks with the argument that delivery was within term, a situation that unfortunately is still going on, especially in the private obstetric practice. At present, in many private facilities in our setting $\mathrm{C}$-section rate is $75 \%$ or higher. This significantly increases the risk for a higher neonatal morbidity and long-term cognitive disorders.

Several studies agree that newborns delivered vaginally at 37-38 weeks of gestation, compared to those delivered at 39-41 weeks, have a higher neonatal mortality, twice as much morbidity, a low Apgar score at birth which is $70 \%$ more common, and admission to the neonatal intensive care unit that is more than two-fold.

A prospective study was conducted at Hospital Italiano de Buenos Aires which showed that neonatal morbidity at 37 weeks was significantly higher among infants delivered by C-section $(7.6 \%)$ compared to those delivered vaginally $(2.1 \%)$ (relative risk [RR]: 3.5, 95\% confidence interval [CI]: 1.50-8.1). ${ }^{1}$

A study included infants born between 37 and 41 weeks of gestation $(n=35539)$ divided into 2 cohorts: early term infants born at 37-38 weeks $(\mathrm{n}=11318)$ and full term infants born at 3941 weeks $(n=24221)$. C-section delivery was higher among early term infants than full term ones (38.3\% versus $31.3 \%, p<0.0001)$. Significant differences were observed in the prevalence of morbidity among infants born at 37-38 weeks compared to those born at 39-41 weeks. The most important differences were admission to the neonatal unit: $9.1 \%$ versus $3.5 \%(p<0.0001)$, hyaline membrane disease: $0.14 \%$ versus $0.007 \%$ $(p<0.0001)$, mechanical ventilation: $0.2 \%$ versus $0.07 \%(p<0.009)$, and continuous positive airway pressure (CPAP):0.11\% versus $0.01 \%(p<0.0001){ }^{2}$

Another study assessed a different aspect: the impact of gestational age on breastfeeding among term infants. A population of 80297 neonates who were exclusively or partially breastfed upon discharge from the hospital were included in the study. A multivariate logistic regression analysis demonstrated that early term infants had a significantly lower likelihood of being breastfed than infants born at 40 weeks (adjusted odds ratio [aOR]: at 38 weeks (aOR: 0.81, 95\% CI: 0.75-0.88), and at 37 weeks (aOR: $0.74,95 \%$ CI: $0.67-0.82){ }^{3}$

However, although these aspects are very important, the most concerning morbidity at present is long-term neurodevelopmental outcome.

Several studies have evaluated the course of fetal nervous system development. It is currently known that in the second half of gestation the brain develops continuously, slowly at first and then at a faster pace. A clear example of this is that at 34 weeks, the brain weighs only $60 \%$ of the weight it reaches between 39 and 41 weeks, and its volume increases five times. ${ }^{4}$ In addition, neurological maturation continues being very active through neurogenesis, synaptogenesis, dendritic arborization, and glial cell proliferation up to 41 weeks of gestation.

Some studies assessed the risk for long-term cognitive disorders among infants born at 3941 weeks and found that risks in this group were significantly lower than among early term infants (37-38 weeks), who showed a worse outcome in relation to growth and education.

Kimberly G. et al. conducted a study in the United States in a population of 128050 infants delivered between 37 and 41 weeks from a normal pregnancy. At 8 years old, academic performance was studied in these children based on their gestational age at birth. The scores in the school performance of children born at 37-38 weeks were significantly worse than of those born at 39-41 weeks. This effect was independent from birth weight and social and economic factors. The authors pointed out that investigators, physicians, and patients should consider these outcomes to identify infants at risk of having a worse academic performance. ${ }^{5}$

There are two recent studies to note, in one of which, school performance was assessed at 
7 years old among children born early term. In comparison with infants born between 39 and 40 weeks, those born between 37 and 38 weeks had significantly lower scores in four out of the five domains under investigation. ${ }^{6}$ The other describes study compared school performance at 5 years old by gestational age at birth. Among those born at 37-38 weeks, $51 \%$ did not reach a good overall performance. ${ }^{7}$

In this situation, a task force made up of several scientific societies from the United States studied this problem and proposed to replace the definition of term pregnancy between 37 and 41 weeks of gestation with a different classification. They recommended replacing this definition with early term (37-38 weeks), full term (39-40 weeks and 6 days), and late term (41 weeks). In addition, they recommended to discourage the use of the label "term pregnancy" to describe all deliveries occurred between 37 and 41 weeks of gestation in the obstetric practice. ${ }^{8}$

This proposal was accepted by the American College of Obstetricians and Gynecologists and the Society for Maternal-Fetal Medicine of the United States, who supported and encouraged the use of the task force denomination with the new labels for gestational age among physicians, investigators, and public health officers to facilitate data submission, high quality health care services, and clinical research. ${ }^{9}$

However, in spite of all these efforts, many obstetricians continue performing C-sections and vaginal deliveries before 39 weeks of gestation, although it is clearly rejected worldwide. In my opinion, we should strongly advocate for an adequate compliance with the current standards based on the evident harm caused by a forced delivery at 37-38 weeks of gestation.

José M. Ceriani Cernadas, M.D. Editor in chief

http: / / dx.doi.org/10.5546/ aap.2017.eng.410

To cite: Ceriani Cernadas JM. Changes in the definition of term infants and their implications in perinatal care. Are they being accomplished? Arch Argent Pediatr 2017;115(5):410-411.

\section{REFERENCES}

1.- Ceriani Cernadas JM, Mariani G, Pardo A, et al. Nacimiento por cesárea al término en embarazos de bajo riesgo: efectos sobre la morbilidad neonatal. Arch Argent Pediatr 2010;108(1):17-23.

2.-Martínez-Nadal S, Demestre X, Raspall F, et al. Morbilidad neonatal en los recién nacidos a término precoz. An Pediatr (Barc) 2014;81(1):39-44.

3.-Lutsiv O, Giglia L, Pullenayegum E, et al. A Population-Based Cohort Study of Breastfeeding According to Gestational Age at Term Delivery. J Pediatr 2013;163(5):1283-8.

4.- Vorh B. Long-term outcomes of moderately pretem, late prterm and early term infants. Clin Perinatol 2013;40(4):73951.

5.- Noble KG, Fifer WP, Rauh VA, et al. Academic Achievement Varies With Gestational Age Among Children Born at Term. Pediatrics 2012;130(2):e257-64.

6.- Chan E, Quigley MA. School performance at age 7 years in late preterm and early term birth: a cohort study. Arch Dis Child Fetal Neonatal Ed 2014;99(6):F451-7.

7.- Quigley MA, Poulsen G, Boyle E, et al. Early term and late preterm birth are associated with poorer school performance at age 5 years: a cohort study. Arch Dis Child Fetal Neonatal Ed 2012;97(3):F167-73.

8.- Spong CV. Defining “Term” Pregnancy Recommendations From the Defining "Term" Pregnancy Workgroup. JAMA 2013;309(23):2445-6.

9. ACOG Committee Opinion No 579: Definition of term pregnancy. Obstet Gynecol 2013;122(5):1139-40. 